\title{
3D Force Sensor Designed Using Pressure Sensitive Electric Conductive Rubber
}

\author{
Tao Liu, Yoshio Inoue, and Kyoko Shibata \\ Department of Intelligent Mechanical System s Engineering \\ Kochi University of Technology \\ 185 Miyanokuchi, Tosayam ada-Cho, Kami-City, Kochi 782-8502, Japan \\ Email: liu tao@kochi-tech.ac.jp
}

\begin{abstract}
To perform 3D ground reaction force measurements with a comfortable imerface for human foot, this paper presents a new 3D force sensor designed using pressure sensitive electric conductive rubber (PSECR), A parallel supp ort flexible mec hanism was firstly proposed for sensing impact forc es and moments Finite element analysis was conducted for op timization design of sensor structure dimensions. Sensitivity of the 3D force sensor was inlp roved by distrib uting sensing cells on the maximum strain positions. A compact electrical bardware system including amp lifiers module, conditioning circ uits and a micro-c 0mputer controller was developed and integrated into the force sensor. Calb ration experiments were conducted, in which a force plate was used as verification measurement device. The experimental results showed that correlation coefficients of two sensor systems for the vertical force and horizontal force measurements were 0.95 and 0.81 respectively.
\end{abstract}

Keywords-3D force sensor; finite element method; human dy namics

\section{IN IRODUC IION}

It is important to identify 3D reaction force distribution produced by hum an body in the study of human-machine inter action and biomechanical analysis [1] [2]. Ground reaction m easurement with force plates has been successfully devoted to perform dynamics analysis of daily behaviors in many environments [3] [4]. However this method needs sizeable operation space and expensive signal processing devices, so it is inefficient in real time controls and is expensive for daily product developments.

Some wearable sensor systems have been developed for applications in human dynamics analysis. Pressure sensors were widely used to estimate the distributed vertical ground reaction forces and determine the loading pattern of the plantar soft tissue in the stance phase of gait [5] [6] [7], but in these systems the effects of shearing forces or friction forces were neglected. Som e silicon sensors recently are developed to measure both compressive and shear forces at the skin-object interface [8] [9], but the force levels of these sensors are limited in the measurements of sm all forces (about 50N).

Two multi-dimensional sensors for human dynamics analysis have been introduced in [10] and [11], but these sensors were made with serial structures, in which each load cell must be strong enough to stand loads originating from nonmeasurement directions. Moreover these serial structure sensors with load-coupling were difficult to be calibrated Liu proposed a six-axis sensor with four six-axis load cells distributed on four support point, but the measurement ranges in the six-axis are never enough for hum an reaction-force measurements [12]. The force sensors with parallel support mechanism developed by Nishiwaki [13] can be used to measure the reaction force during human walking and control humanoid robots' zero m om ent point (ZMP). This sensor is, however, a little ambitious in its claim that the sensor prototype can be universally used in experim ents with humanoid robot and humans, because its weight of $700 \mathrm{~g}$ and height of $35 \mathrm{~mm}$ was not appropriate to reduce the disturbance of human walking.

We are developing a wearable 3D force senor to measure ground reaction force during hum an walking. In our prior study, we had made a multi-axial force sensor to measure 3 -axial ground reaction forces and coordinates of center of pressure, when fix ed under a specially designed shoe [14]. How ever, its hard interface and weight load for the foot affected human normal walking in our ex perimental study. As shown in Fig. 1, a sensor matrix will be constructed to make a $3 \mathrm{D}$ force sensor interface, which can be integrated into a flexible material, so the sensor system can perform 3D ground reaction force measurements with a comfortable interface for foot. A new design of $3 \mathrm{D}$ force sensor is presented in this paper.

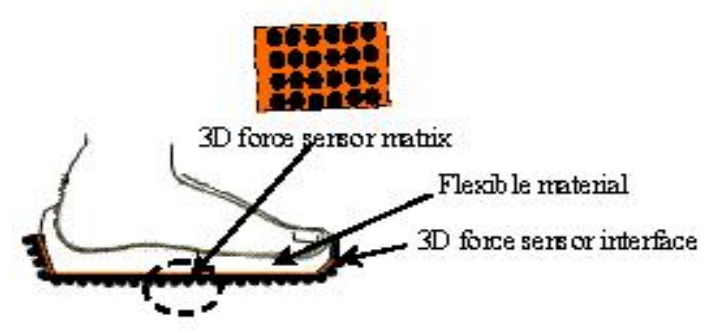

Fig. 1 A 3D reaction force measurement system 


\section{METHODS AND MATERIAL}

\section{A. Cell of $3 D$ Force Sensor}

Sensing cells of 3D sensor were designed using pressure sensitive electric conductive rubber (PSECR) which has been used for measuring pressure force distribution. PSECR has been developed for the sheet-switch of the electronic circuits, and has a unique property in that it conducts electric current only when compressed, and acts as an insulator when the pressure is released. The material properties of PSECR are given in Table I.

Table I Material properties of PSECR

\begin{tabular}{cc} 
Color & gray-black \\
Tensile strength & $1.86 \mathrm{MPa}$ \\
Elongation at break & $220 \%$ \\
$100 \%$ modulus & $0.86 \mathrm{MPa}$ \\
Tear resistance & $7 \mathrm{kN} / \mathrm{m}$ \\
Hardness in Durometer & 50 \\
\hline
\end{tabular}

Single sensing cell constructed by five sensing points can measure three-directional forces. As shown in Fig. 2, five pectinate circuits were made to construct a sensing matrix. The interface of the sensor is developed to transform input 3directional forces into pressure forces on the base plate (Fig. 3).

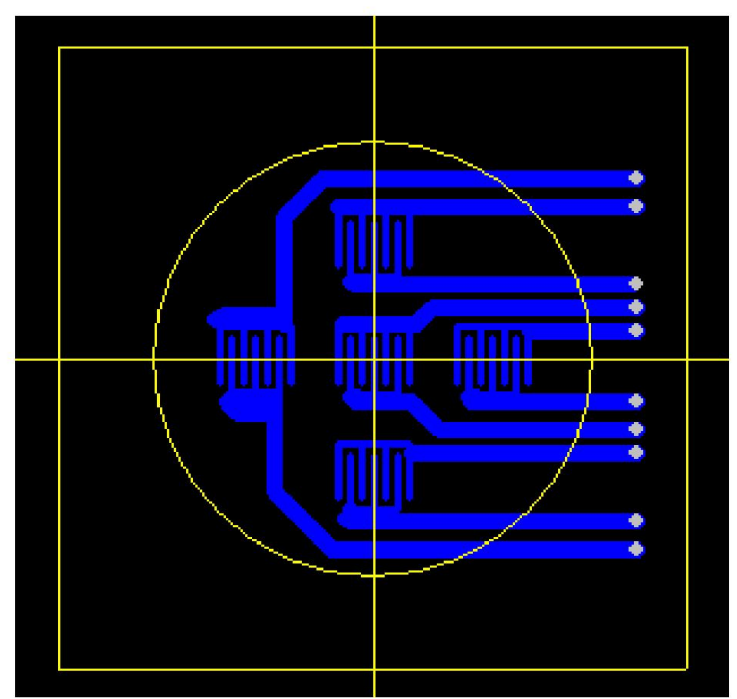

Fig. 2 Pectinate circuit
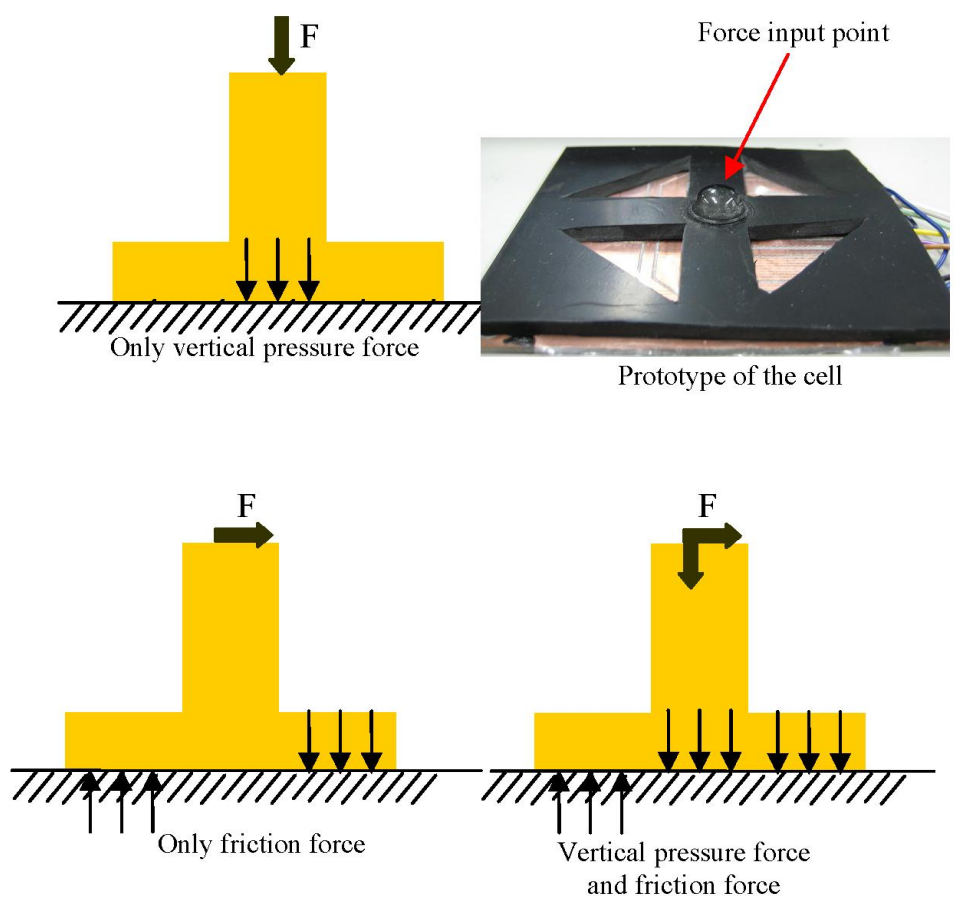

Fig. 3 Cell of 3D force sensor

\section{B. 3D Force Sensor}

Serial and parallel mechanisms are two common designs used in force sensors development. Because serial structure has problem of low stiffness, parallel structure is adopted in our design, under the consideration of needing high stiffness.

As shown in Fig. 4, the developed sensor is composed of a bottom plate and four cells of 3D force sensor. The new sensor was designed to measure three-directional forces and moments. When the forces and the moments are applied on the bottom plane, they are transferred to the four support cells. Translational forces are transferred to all the cells, and are measured by the sensing points attached on the cells of 3D force sensors. Three-direction forces on the four support points can be measured respectively. The $\mathrm{X}$-axial shearing forces are defined as $F_{X 1}, F_{X 2}, F_{X 3}$ and $F_{X 4}$. Similarly, the $Y$-load cell measures shearing forces of $F_{Y 1}, F_{Y 2}, F_{Y 3}$ and $F_{Y 4}$, and the $Z$ load cell measures pressure forces of $F_{Z 1}, F_{Z 2}, F_{Z 3}$ and $F_{Z 4}$. Based on these measured values, the three-directional forces and moments can be calculated as following equations.

$$
\begin{gathered}
F_{x}=F_{x 1}+F_{x 2}+F_{x 3}+F_{x 4} \\
F_{y}=F_{y 1}+F_{y 2}+F_{y 3}+F_{y 4} \\
F_{z}=F_{z 1}+F_{z 4}+F_{z 2}+F_{z 3} \\
M_{x}=\left(F_{z 1}+F_{z 2}-F_{z 3}-F_{z 4}\right) L / 2
\end{gathered}
$$




$$
\begin{aligned}
& M_{y}=\left(F_{z 1}+F_{z 4}-F_{z 2}-F_{z 3}\right) L / 2 \\
& M_{z}=\left(F_{x 4}+F_{x 3}+F_{y 3}+F_{y 2}-\right. \\
& \left.F_{x 1}-F_{y 1}-F_{x 2}-F_{y 4}\right) L / 2
\end{aligned}
$$

\section{Dimension Optimization}

Figure 4 shows detail distribution of the sensing cell. Four cells of 3D force sensor were attached on four support points to measure three-axial translational force. In order to obtain high sensitivity, the sensing points of each cell should be distributed on the position where maximum strains occur. The finite element numerical package ANSYS 8.1 provided by USA ANSYS co., was used to perform the static analysis of the sensor structure. As shown in Fig. 5, 3D FEA model was built for optimal analysis. Based on a defined sensitivity limitation for the sensing material, optimal dimensions of the sensor system were obtained from FEA simulation results. Figure 6 shows a sample of the results of the static analysis for the sensor structure.

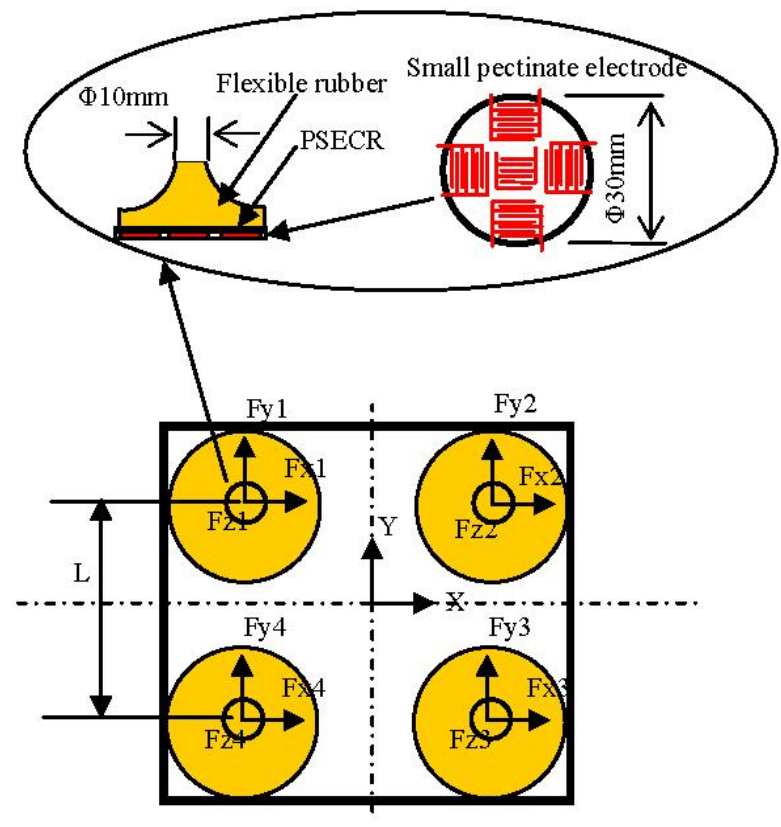

Fig. 4 Mechanism of developed 3D force Sensor

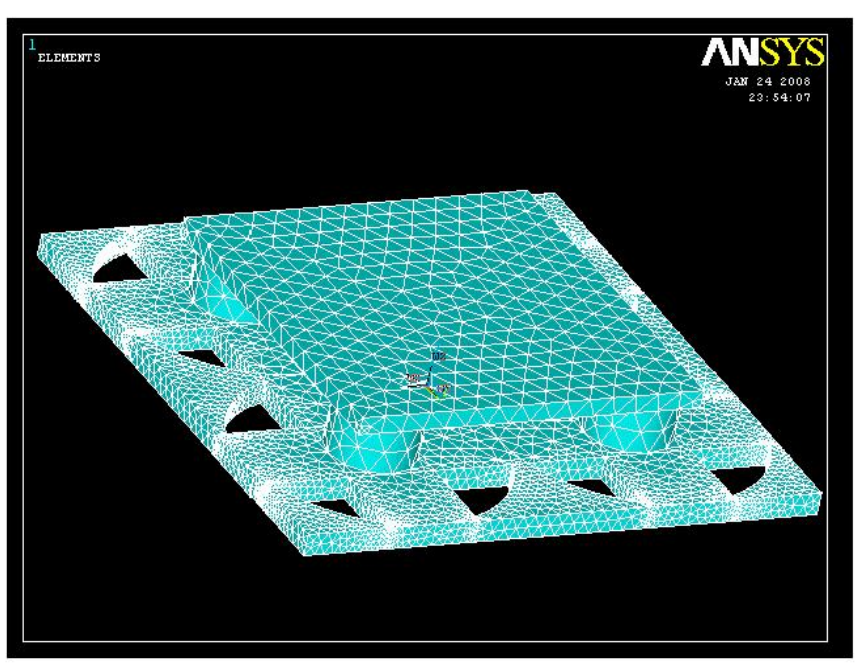

Fig. 5 FEA model in ANSYS

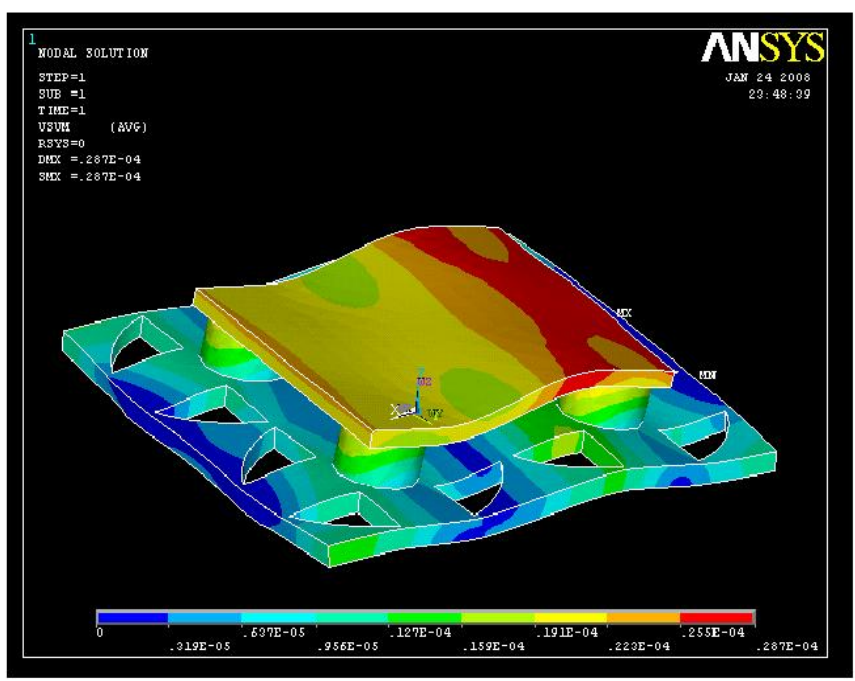

Fig. 6 A sample analysis result

\section{Electrical system design of the new sensor}

As shown in Fig. 7, an integrated electrical system was developed and incorporated into the force sensor. The 3D forces applied on the flexible interface are converted to the resistance changes of sensing points using PSECR. Then the resistance changes are converted to the voltage signals by the conditioning modules, and are amplified by the amplifier modules. The amplified voltage signals are input into $\mathrm{PC}$ after A/D conversion using a micro-computer H8 3964. Since 12 sensing points were used (four groups for X-direction, four groups for Y- direction force and another four groups for Zdirection forces), there were 12 channels of the voltage signals. Fig. 8 gives a simple explanation of the hardware system of the 3D force sensor, and a data processing program developed using MATLAB specially was used to sample the 12 channels of the voltage signals, and calculate the reaction forces and moments. 


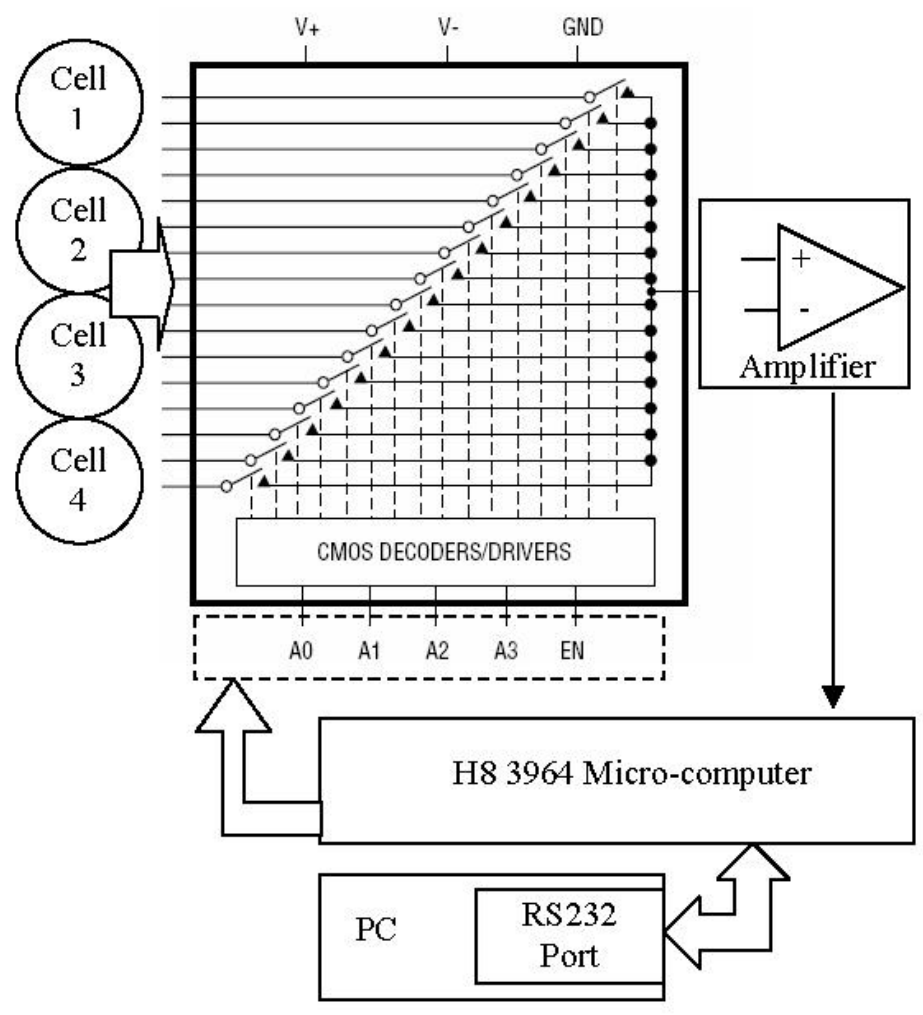

\section{A. Calibration}

.In order to calibrate the developed sensor system, EFP-S2KNSA12, a six-dimensional force/moment sensor was used as verification measurement device. These two sensor systems worked in a synchronized mode. The experimental system was established as shown in Fig. 9.

It was mentioned above that there were 12 channels of the voltage signals $\mathrm{X}_{1}(\mathrm{i}=1, \ldots, 12)$. Based on $\mathrm{X}_{1}, \ldots \mathrm{X}_{12}$, so to calibrate the sensor system, the forces and the moments can be calculated as the following equations.

$$
\begin{gathered}
F_{x}=\sum_{i=9}^{12} A_{i} X_{i} \\
F_{y}=\sum_{i=5}^{8} A_{i} X_{i} \\
F_{z}=\sum_{i=1}^{4} A_{i} X_{i} \\
M_{x}=\left(\sum_{i=2}^{3} A_{i} X_{i}-\sum_{i=1}^{4} A_{i} X_{i}\right) \frac{L}{2} \\
M_{y}=\left(\sum_{i=3}^{4} A_{i} X_{i}-\sum_{i=1}^{2} A_{i} X_{i}\right) \frac{L}{2} \\
M_{z}=\left(\sum_{i=5}^{12}(-1)^{i+1} A_{i} X_{i}\right)\left(\frac{L}{2}\right)
\end{gathered}
$$

where the $X_{i}$ are the sensing cells' conditioning outputs, and $A_{i}$ $(i=1, \ldots, 12)$ are calibration coefficients for each sensing cells.

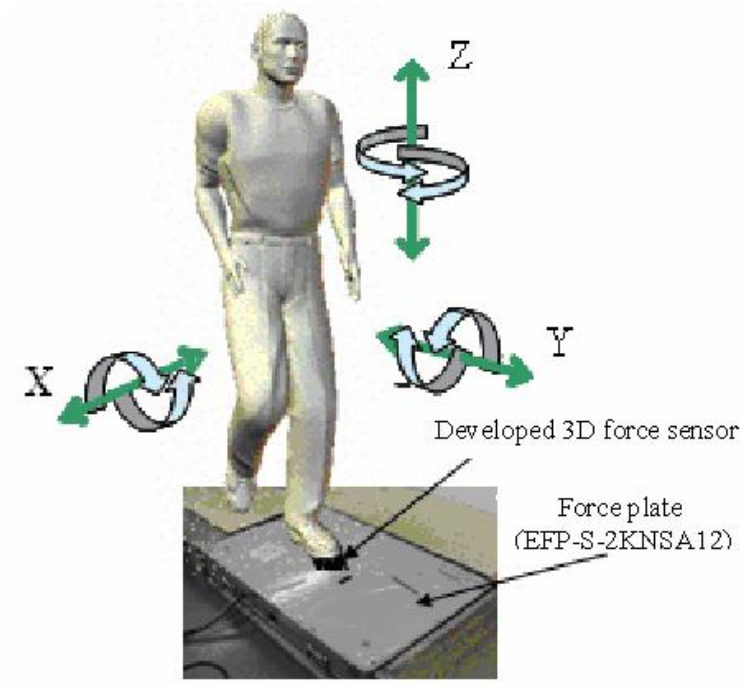

Fig. 9 Calibration experimental system 
Multiple regression analysis was used for the calculation of the calibration coefficients. In this study, we finished multiple regression analysis of the data using a statistical software of SPSS 11.0J. The results of the multiple regression analysis $\left(A_{i}\right)$ were used for the next experimental study of the developed sensor.

\section{B. Ground reaction force measurement}

Force plate (EFP-S-2KNSA12) was used as the verification sensor to calibrate the develop sensor. In our experiment, these two sensor systems worked in the synchronized mode. Measured data from these two sensors were sampled at the same frequency, and were compared to verify the developed sensor system. The correlation coefficient was used as a measure of the association between two results of the two sensor systems, and the correlation coefficients for the vertical force and horizontal force measurements were 0.95 and 0.81 respectively.

\section{CONCLUSION}

A new 3D force sensor developed using pressure sensitive electric conductive rubber is proposed for measuring threedirectional reaction forces and three-directional moments in human dynamics analysis. Finite element method was adopted to optimize the mechanism structure dimensions, and improve the sensitivity of the force sensor by distributing the sensing cells on the maximum strain positions. A compact electrical hardware system including amplifiers module, conditioning circuits and a micro-computer controller was developed for the force sensor system. The calibration experiments were performed to calculate calibration coefficients by using the multiple regression analysis method and test linear property of the sensor. In the next step of human dynamics analysis, the $3 \mathrm{D}$ force sensor will be used for ground reaction forces measurements in human dynamic analysis.

\section{REFERENCES}

[1] Gerald F. Harris, Peter A. Smith, "Human Motion Analysis," Reading, IEEE, Inc., pp 12-30, 1996.

[2] Mehmet Engin, Alparslan Demirel, Erkan Zeki Engin, Musa Fedakar, "Recent developments and trends in biomedical sensors," Measurement, 37, pp. 173-188, 2005.

[3] Kim C.M, Eng J.J, "Magnitude and pattern of 3D kinematic and kinetic gait profiles in persons with stroke: relationship to walking speed," Gait and Posture, vol.20, pp.140-146, 2004

[4] Casadio M, Morasso P.G, Sanguineti V, "Direct measurement of ankle stiffness during quiet standing: implications for control modelling and clinical application," Gait and Posture, Vol.21, pp.410-424, 2005.

[5] Yoshio INOUE, Takuya MATSUDA, Kyoko SHIBATA, "Estimation of vertical reaction force and ankle joint moment by using plantar pressure sensor," JSME, Symposium on human dynamics, pp.57-62, 2003. (In Japanese).

[6] Ledoux W.R, Hillstrom H.J, "The distributed plantar vertical force of neutrally aligned and pes planus feet," Gait and Posture, Vol.15, pp.1-9, 2002 .

[7] Faivre A, Dahan M, Parratte B, Monnier G, "Instrumented shoes for pathological gait assessment," Mechanics Research Communications, Vol.31, pp.627-632, 2004.

[8] Beccai L, Roccella S, Arena A, Valvo F, Valdastri P, Menciassi A, Carrozza M.C, Dario P, "Design and fabrication of a hybrid silicon three-axial force sensor for biomechanical applications," Sensors and Actuators A: Physical, Vol.120, pp.370-382, 2005.

[9] Valdastri P, Roccella S, Beccai L, Cattin E, Menciassi A, Carrozza M.C, Dario P, "Characterization of a novel hybrid silicon three-axial force sensor," Sensors \& Actuators: A. Physical, Vol.123-124, pp.249-257, 2005

[10] Chao L.-P, Yin C.-Y, "The six-component force sensor for measuring the loading of the feet in locomotion," Materials and Design, vol.20, pp.237-244, 1999.

[11] Vazsonyi E, Adam M, Ducso Cs, Vizvary Z, Toth A.L, Barsony I, "Three-dimensional force sensor by novel alkaline etching technique," Sensors and Actuators: A. Physical, Vol.123-124, pp.620-626, 2005.

[12] S. A. Liu, "A novel six-component force sensor of good measurement isotropy and sensitivities," Sensor and Actuators, pp.223-230,2002.

[13] Koichi Nishiwaki, "A six-axis force sensor with parallel support mechanism to measure the ground reaction force of humanoid robot," Proceeding of the 2002 IEEE international conference on robotics and automation, pp.2277-2282, 2002.

[14] Tao Liu, Yoshio Inoue, Kyoko Shibata, Yohei Yamasaki, Masafumi Nakahama, "A Six-dimension Parallel Force Sensor for Human Dynamics Analysis," Proceedings 2004 IEEE International Conference on Robotics, Automation and Mechatronics, pp. 208-212, 2004 